\title{
Spectrum of disease and outcome of primary amputation for diabetic foot sepsis in rural KwaZulu-Natal
}

\author{
S Cheddie, ${ }^{1}$ CG Manneh, ${ }^{2}$ B Pillay ${ }^{3}$ \\ ${ }^{1}$ Head Clinical unit: Surgery, Madadeni Hospital, Honorary lecturer UKZN \\ ${ }^{2}$ Medical Officer: Surgery, Madadeni Hospital \\ ${ }^{3}$ Head of Vascular Surgery unit: Inkosi Albert Luthuli Central Hospital, Honorary lecturer UKZN
}

Corresponding author: Shalen Cheddie (scheddie@gmail.com)

\begin{abstract}
Background: Primary amputation (stump closure) for diabetic foot sepsis is perceived to have a higher re-amputation rate due to stump sepsis. A guillotine amputation with elective stump closure is widely practised due to the lower risk of stump sepsis and re-amputation.

Aims: To provide an epidemiological analysis of the spectrum of disease and outcomes of primary amputation for diabetic foot sepsis in a regional rural hospital.

Methods: A prospective cohort study of 100 patients who underwent surgery for diabetic foot sepsis over a 5-year period was undertaken at Madadeni Provincial Hospital, in northern KwaZulu- Natal. Demographic data, co-morbid profile, radiographic features, anatomical level of vascular occlusion and type of surgery performed were recorded. The Wagner classification (Wag) was used to classify disease severity. Outcome measures included length of hospital stay, in-hospital mortality and re-amputation rates.

Results: Of the 100 patients, females $(n=50)$ accounted for $50 \%$ of admissions. The median age was 61 years (range: 29 to 80 years). Most patients presented with advanced disease: Wag 5, $\mathrm{n}=71(71 \%)$; Wag 4, $\mathrm{n}=20(20 \%)$; Wag 3, $\mathrm{n}=7(7 \%)$; Wag $2, \mathrm{n}=2(2 \%)$. The anatomic levels of vascular occlusion comprised: aortoiliac disease $\mathrm{n}=2(2 \%)$, femoropopliteal $n=21(21 \%)$ and tibioperoneal disease $n=77(77 \%)$. The following surgical procedures were undertaken: above knee amputation (AKA), $\mathrm{n}=35(35 \%)$; below knee amputation (BKA), $\mathrm{n}=46(46 \%)$; transmetatarsal amputation (TMA), $\mathrm{n}=8(8 \%)$; toe ectomy, $\mathrm{n}=8(8 \%)$ and debridement, $\mathrm{n}=3(3 \%)$. The re-amputation rate to above knee amputation was $\mathrm{n}$ $=2 / 46(4.3 \%)$. All AKA stumps healed completely. The overall in-hospital mortality was $\mathrm{n}=7(7 \%)$ and median length of hospital stay was $7.8 \pm 3.83$ days.

Conclusion: Most patients present with advanced disease requiring a major amputation. A definitive one stage primary amputation is a safe and effective procedure for diabetic foot sepsis with distinct advantages of a short hospital stay, low reamputation rates and mortality. A guillotine amputation should be reserved for physiologically unstable patients.
\end{abstract}

Keywords: amputation; diabetic foot; guillotine; re-amputation

S Afr J Surg 2018;56(3)

http://dx.doi.org/10.17159/2078-5151/2018/v56n3a2486

\section{Introduction}

Diabetic foot sepsis is a major burden of disease and carries a significant morbidity and mortality. Foot complications are among the most serious and costly complications of diabetes mellitus, with ulceration increasing worldwide; over $80 \%$ of foot and lower limb amputations are preceded by a foot ulcer. Worldwide in 2003 there were 194 million people between the ages 20-79 with diabetes; currently the estimate is that among adults the prevalence of diabetes is expected to reach 333 million by $2025 .^{1}$

The major concern with a primary BKA in the setting of diabetic foot sepsis is infection of the below knee stump. This complication is estimated at $25 \%$ and will necessitate an AKA. A landmark local study by Desai and colleagues demonstrated that an initial guillotine amputation (no closure of skin flaps) done in the acute setting followed by a revision amputation in seven days yielded better results with respect to stump sepsis rates and thus lead to a lower AKA rate. Patients undergoing a primary BKA (skin and muscle flap closure) had a reamputation (conversion to above knee level) rate of $33.3 \%$ while those undergoing a guillotine amputation followed by a revision within seven days had a re-amputation rate of $7.7 \%{ }^{2}$ In view of these findings, it has become common practice to adopt a two stage approach to amputations in the setting of extremity sepsis. 
The aim of this study was to provide an epidemiological analysis of patients presenting with diabetic foot sepsis, and to re-evaluate the role of primary amputation for diabetic foot sepsis exclusively with respect to specific outcome measures mainly, the re-amputation rate.

\section{Methods}

The study was approved by the Biomedical Research Ethics Committee of University of KwaZulu-Natal (BE: 335/17). A prospective cohort study of 100 patients was undertaken at a regional rural hospital in KwaZulu-Natal (KZN). All patients undergoing surgery for diabetic foot sepsis from January 2013 to March 2017 were included in the data analysis. BKA was performed if 1) sepsis extended beyond the fore-foot and below the mid-tibia level and 2) a palpable popliteal pulse was present. An AKA was performed if there was 1) contracture of the knee joint, 2) absent popliteal pulse and 3) sepsis extending proximal to the mid-tibia level. Physiologically stable patients who required a BKA underwent a primary amputation and those who were physiologically unstable underwent a guillotine amputation. Patients who underwent a guillotine amputation were excluded from the study. All patients were given therapeutic intravenous amoxicillin-clavulanic acid for 5 days from admission. Surgery was performed within 24 hours of admission.

\section{BKA technique}

A long posterior flap incision is created and the tibia is divided $1 / 3$ rd the distance from the tibial tuberosity to the medial malleolus. The fibula is divided $2 \mathrm{~cm}$ higher than the tibia. The bone edges are bevelled. The anterior and posterior tibial vessels are divided and ligated with absorbable suture and the tibial nerve is stretched, ligated and divided. Haemostasis is achieved with suture ligation only and a washout of the stump is done with sterile water. The fascia is closed with interrupted absorbable sutures in order to allow a tension free closure. The skin edges are approximated with interrupted nylon sutures which are spaced $2 \mathrm{~cm}$ apart and the wound is dressed with paraffin gauze, dressing gauze and crepe bandage.

Variables included in the data analysis were demographic details: age; gender and race. Co-morbidities - renal failure (as defined by serum Creatinine $>1.5$ time normal) and diabetic ketoacidosis - were recorded. The severity of disease was classified according to the Wagner classification. ${ }^{3}$ Radiographic features, i.e. normal, osteitis and gas gangrene were recorded. The anatomical level of vascular occlusion (tibioperoneal, femoropopliteal and aortoiliac disease) and the type of surgery (debridement, toe ectomy, TMA, BKA and AKA) were recorded. Outcome measures included reamputation, re-debridement rates, length of hospital stay and in-hospital mortality. Simple statistical analysis, including median, mean, range and standard deviation for continuous variables, was done using GraphPad Software (LaJolla, USA).

\section{Results}

Of the 100 patients, females $(n=50)$ accounted for $50 \%$ of admissions. The median age was 61 years (range: 29 to 80 years) and the majority of patients were African, $\mathrm{n}=89$ (89\%). Only 1 patient presented with diabetic ketoacidosis and $18(18 \%)$ presented with renal failure. Most patients presented with advanced disease: Wag 5, $\mathrm{n}=71$ (71\%); Wag 4, $\mathrm{n}=20$ (20\%); Wag 3, $\mathrm{n}=7$ (7\%); Wag 2, $\mathrm{n}=2$ (2\%). The levels of vascular occlusion included: aortoiliac disease, $\mathrm{n}=2(2 \%)$; femoropopliteal disease, $\mathrm{n}=21(21 \%)$; and tibioperoneal disease $n=77$ (77\%). Radiographic features included normal findings. $\mathrm{n}=65(65 \%)$; gas visible on X-Ray, $\mathrm{n}=14$ (14\%); osteitis $\mathrm{n}=13(13 \%)$ (see Table 1).

The following surgical procedures were done: AKA, $\mathrm{n}=35(35 \%)$; BKA, $\mathrm{n}=46(46 \%)$; TMA, $\mathrm{n}=8(8 \%)$; toe ectomy, $\mathrm{n}=8(8 \%)$; and debridement, $\mathrm{n}=3(3 \%)$ (see Table $2)$. The re-amputation rate to above knee amputation was $\mathrm{n}=2 / 46(4.3 \%)$. All AKA stumps healed completely. The overall in-hospital mortality was $n=7(7 \%)$. Five of the seven mortalities were as a result of myocardial infarction; one patient succumbed to a major stroke and one patient

\begin{tabular}{lc}
$\begin{array}{l}\text { Table 1: Demographic profile and spectrum of disease severity } \\
\text { (Wagner/Radiographic abnormalities) }\end{array}$ \\
\hline \multicolumn{1}{l}{ Variables } & \multicolumn{1}{l}{ Median (range); n (\%) } \\
\hline Demographic Profile & $61(29-80)$ \\
Ages & $50(50 \%)$ \\
Males & $50(50 \%)$ \\
Female & \\
Co-morbidity & $18(18 \%)$ \\
Renal failure & $1(1 \%)$ \\
DKA & \\
Severity & $71(71 \%)$ \\
Wagner 5 & $20(20 \%)$ \\
Wagner 4 & $7(7 \%)$ \\
Wagner 3 & $2(2 \%)$ \\
Wagner 2 & \\
Radiographic abnormalities & $65(65 \%)$ \\
Normal & $13(13 \%)$ \\
Osteitis & $14(14 \%)$ \\
Gas visible on & \\
X-Ray &
\end{tabular}

Table 2: Anatomical levels of vascular occlusion and types of amputation

\begin{tabular}{lcccc}
\hline & $\begin{array}{c}\text { Tibioperoneal } \\
\mathbf{n}(\mathbf{\%})\end{array}$ & $\begin{array}{c}\text { Femoropopliteal } \\
\mathbf{n}(\mathbf{\%})\end{array}$ & $\begin{array}{c}\text { Aortoiliac } \\
\mathbf{n}(\%)\end{array}$ & $\begin{array}{c}\text { Total } \\
\mathbf{n}(\%)\end{array}$ \\
\hline Toe & $7(87.5)$ & $1(12.5)$ & 0 & $8(8)$ \\
ectomy & & & & \\
TMA & $8(100)$ & 0 & 0 & $8(8)$ \\
BKA & $43(93.5)$ & $3(6.5)$ & 0 & $46(46)$ \\
AKA & $15(42.9)$ & $18(51.4)$ & $2(5.7)$ & $35(35)$
\end{tabular}




\begin{tabular}{|c|c|c|c|}
\hline & $\begin{array}{c}\text { Re-amputation } \\
\text { n (\%) }\end{array}$ & $\begin{array}{c}\text { Length of Stay } \\
\text { (days) }\end{array}$ & $\begin{array}{c}\text { Mortality } \\
\text { n (\%) }\end{array}$ \\
\hline $\begin{array}{l}\text { BKA } \\
(n=46)\end{array}$ & $2(4)$ & $8.56 \pm 4.15$ days & $3(6.6)$ \\
\hline $\begin{array}{l}\text { AKA } \\
(n=35)\end{array}$ & 0 & $8.13 \pm 3.89$ & $4(11.4)$ \\
\hline
\end{tabular}

had systemic sepsis. The median length of hospital stay was $7.8 \pm 3.83$ days (see Table 3). Of the 46 BKA and 35 AKA patients, only 10 from each group were discharged to our physiotherapy department. All 20 patients were ambulant on prosthesis at a median of 6 months from discharge.

\section{Discussion}

Guillotine amputation for diabetic foot sepsis followed by elective stump closure is widely practiced. ${ }^{2,45-8}$ The concern with primary amputation has been the high risk of stump sepsis, necessitating re-amputation to the above knee level which has a major impact on rehabilitation and quality of life. In KwaZulu-Natal province, routine guillotine amputation has been supported by a local study which showed that patients undergoing a primary BKA had a higher re-amputation (to above knee level) than patients undergoing guillotine amputation followed by a revision amputation. However, this study had a mixed cohort of patients with extremity sepsis and a minority with diabetes mellitus (38.4\%). All patients who had a failed primary BKA and re-amputation to the above knee level had either aortoiliac or femoropopliteal artery occlusive disease which would have been a major risk factor for stump failure due to ischemia. ${ }^{2}$

Mcintyre and colleagues have also shown that a primary amputation in the setting of foot sepsis in a mixed cohort has a lower success rate than a staged amputation (78\% vs. $97 \%$ ) and conversion from BKA to AKA was $11 \%$ in the primary amputation group. ${ }^{4}$ Furthermore, a revision to below knee level after a guillotine amputation has been shown to have a failure rate of $5.9 \%$ vs $33 \%$ when a primary BKA was done for diabetic foot sepsis; the cause of stump failure was attributed to stump sepsis. ${ }^{5}$ Fisher and colleagues have demonstrated a stump sepsis rate of $21 \%$ after a primary BKA for extremity sepsis in a mixed patient cohort, and no stump sepsis following a guillotine amputation with secondary stump closure. ${ }^{6}$

Aulivola et al. have shown the conversion rate of BKA to AKA for stump failure in a mixed cohort was $9.4 \%$, with no difference noted in diabetic and non-diabetic patients. ${ }^{7} \mathrm{~A}$ Cochrane eview has also shown that a guillotine amputation with elective stump closure has better wound healing rates than a primary amputation. ${ }^{8}$

In our study, primary amputations were performed at the below knee and above knee levels. The BKA conversion rate to AKA was $4.3 \%$ and the success rate of a primary AKA was $100 \%$. These results show that primary amputation is safe and effective for the management of diabetic foot sepsis, even in the context of clinically advanced disease. Most patients present with advanced disease (Wag 5 [71\%]) which may be attributed to the rural setting of the study where delays in access to health care facilities are common. Wong et al. have shown a primary BKA success rate of $73.5 \%$ in diabetic foot sepsis patients only. ${ }^{9}$ Viswanathan et al. reported a reamputation rate of only $3 \%$ in a study of 405 diabetic patients in 3 developing countries. ${ }^{10}$

Predictors of wound healing including angiography have not been shown to be completely reliable. Even the presence of a popliteal pulse will only yield a $50 \%$ probability of stump healing. ${ }^{11}$ A study by O'Dwyer had shown that a palpable popliteal pulse was associated with a $90 \%$ healing rate for BKA while the absence of a femoral pulse was associated with a BKA failure rate of $79 \% .{ }^{12}$ Currently the Transcutaneous Oxygen Pressure (TCPO2) measurement is the most reliable test showing an accuracy of $90 \%$ in predicting wound healing. ${ }^{11}$ TCPO2 is, however, unfortunately unreliable in the context of sepsis, where oedema affects oxygen diffusion. ${ }^{11}$ This test is not routinely available at all regional hospitals and patients in KZN can only access it at a solitary vascular laboratory facility. This places a major demand on resources including transportation costs and delays in executing definitive management, especially from rural areas. Cognisance of the abovementioned limitations has led to a primary amputation being standard practice at the authors' centre. A guillotine amputation is still indicated for physiologically unstable patients who require source control of sepsis. This technique is quick and easy to perform with minimal blood loss. A formal BKA can be undertaken once the patient has been optimised and there is resolution of limb oedema and cellulitis.

Underlying peripheral vascular disease often accompanies diabetic foot sepsis in about $50 \%$ of cases. ${ }^{13} \mathrm{~A}$ full neurological assessment was not performed in this study. In diabetic subjects, peripheral arterial disease more frequently affects below-the-knee vessels such as the tibial and peroneal arteries and is symmetric, multi-segmental while the collateral vessels can also be affected by stenosis. ${ }^{14}$ Our study has shown that all patients presented with peripheral arterial disease, i.e. underlying tibioperoneal disease (77\%). Patients presenting with diabetic foot sepsis with a palpable popliteal pulse underwent a primary BKA if sepsis was spreading beyond the transmetatarsal level. An AKA was done if the popliteal pulse was absent. Adhering to this policy, $93.5 \%$ of primary BKAs were performed in patients with tibioperoneal disease. The low re-amputation rate of $4.3 \%$ may be attributed to the good blood supply as demonstrated by a palpable popliteal pulse. This may imply that the reason for stump failure in the postoperative period may be due to ischemia rather than stump sepsis. Wong et al. have shown that an absent popliteal pulse was a significant risk factor for poor outcomes following BKA in diabetic foot sepsis. ${ }^{9}$

Studies have shown that the mean hospital stay following surgery for diabetic foot sepsis is 36.24 days which highlights the morbidity of this disease. The mortality rate of $32 \%$ was 
significantly associated with the grade of disease (Wagner's classification $>4$ ), i.e. worsening severity. ${ }^{15}$ In our study, the median length of hospital stay was $7.8 \pm 3.83$ days and the in-hospital mortality was $7 \%$. The low mortality rate may be attributed to the low co-morbidity rate of renal failure $(18 \%)$ and diabetic ketoacidosis (1\%) in our cohort. The early initiation of broad spectrum antibiotics and early amputation (within 24 hours of admission) may have also contributed to the excellent postoperative outcomes. Physiologically unstable patients who underwent a guillotine amputation were also excluded from the study. The aggressive use of physiotherapy in conjunction with occupational therapy in the early postoperative setting (day one) has resulted in early mobilisation and subsequent hospital discharge.

\section{Limitations}

The rural setting of the study with a predominantly African cohort may limit the generalisability of the findings to the general population. The amputations were performed by a single consultant surgeon with a dedicated assistant, and thus the results may not be attainable at all centres. Limited access to TCPO2 and Doppler studies precluded an accurate assessment of the preoperative vascular status. An accurate assessment of time to ambulation was limited by the stepdown of patients to their base hospitals for rehabilitation and subsequent loss to follow-up.

\section{Conclusion}

Most patients with diabetic foot sepsis in the rural setting of KwaZulu-Natal present with advanced disease requiring a major amputation. A definitive one stage primary amputation is a safe and effective procedure for diabetic foot sepsis, and is associated with a short duration of hospital stay, low re-amputation rates, and mortality. A guillotine amputation should be reserved for physiologically unstable patients.

\section{Conflict of interest:}

The authors do not have any conflict of interest to share.

\section{REFERENCES}

1. Clarke A. Avoiding foot complications in diabetes. CME 2010;28(4):181-5.
2. Desai Y, Robbs JV, Keenan JP. Staged below knee amputation for septic peripheral lesions due to ischaemia. $\mathrm{Br} \mathrm{J}$ Surg. 1986;73:392-4. Available from: https://doi.org/10.1002/ bjs. 1800730527

3. Oyibo SO, Jude EB, Tarawneh I, et al. A comparison of two diabetic foot ulcer classification systems: the Wagner and the University of Texas wound classification systems. Diabetes Care. 2001;24:84-8. Available from: https://doi.org: 10.2337/ diacare.24.1.84

4. Mcintyre KE Jr, Bailey SA, Malone JM, et al. Guillotine amputation in the treatment of Nonsalvageable Lower-extremity infections. Arch Surg. 1984;119(4):450-3. Available from: http:// dx.doi.org :10.1001/archsurg.1984.01390160080016

5. Scher KS, Steele FJ. The septic foot with diabetes. Surgery. 1988;104(4):661-6.

6. Fisher DF Jr, Clagget GP, Fry RE, et al. One stage versus two stage amputation for wet gangrene of the lower extremity: a randomised study. J Vasc. 1988;8(4):428-33. Available from: https://doi.org/10.1067/mva.1988.avs0080428

7. Aulivola B, Hile CN, Hamdan AD, et al. Major lower extremity amputation: Outcome of a modern series. Arch Surg. 2004;139:395-9. Available from: https://doi.org/10.1016/j. jvs.2004.05.018

8. Tisi PV; Than MM. Type of incision for below knee amputation. Cochrane Database Syst Rev. 2014;(4):CD003749. ISSN: 1469-493X

9. Wong LK, Nather A, Liang S, et al. Clinical Outcomes of Below Knee Amputations in Diabetic Foot Patients. Ann Acad Med Singapore. 2013;42:388-94.

10. Viswanathan V, Wadud JR, Madhavan S, et al. Comparison of post amputation outcome in patients with type 2 diabetes from specialised foot care centres in three developing countries. Diabetes Res Clin Pract. 2010;88:146-50. Available from: https://doi.org/10.1016/j.diabres.2010.02.015

11. Robbs JV. Lower limb amputation for ischaemia with special reference to the diabetic patient. CME. 2010;28(4):164-70.

12. O'Dwyer KJ, Edwards MH. The association between lowest palpable pulse and wound healing in below knee amputations. Ann R Coll Surgeons Engl. 1985;67(4):232-4.

13. Tudhope L. Principles of management of vascular problems in the diabetic foot. CME. 2010:28(4):158-63.

14. Aiello A, Anichini R, Brocco E, et al. Treatment of peripheral arterial disease in diabetes: A consensus of the Italian Societies of Diabetes (SID, AMD), Radiology (SIRM) and Vascular Endovascular Surgery (SICVE). Nutrition, Metabolism and Cardiovascular Diseases. 2014; 24:355-69. Available from: http://dx.doi.org/10.1016/j.numecd.2013.12.007

15. Chalya PL, Mabula JB, Dass RM. Surgical management of diabetic foot ulcers: A Tanzanian university teaching hospital experience. BMC Research Notes. 2011;4:36. Available from: https://doi.org/10.1186/1756-0500-4-365 